Pembrolizumab Plus Ipilimumab as Second-Line Therapy for Advanced NSCLC

\title{
Pembrolizumab in Combination with Ipilimumab as Second-Line or Later Therapy for Advanced Non-Small-Cell Lung Cancer: KEYNOTE-021 Cohorts D and H
}

Running title: Pembrolizumab Plus Ipilimumab as Second-Line Therapy for Advanced NSCLC

M. A. Gubens, ${ }^{a}$ L. V. Sequist,${ }^{b}$ J. P. Stevenson, ${ }^{\mathrm{c}}$ S. F. Powell, ${ }^{\mathrm{d}}$ L. C. Villaruz, ${ }^{\mathrm{e}}$ S. M. Gadgeel,${ }^{\mathrm{f}}$ C. J. Langer, ${ }^{\mathrm{g}}$ A. Patnaik, ${ }^{\mathrm{h}}$ H. Borghaei, ${ }^{\mathrm{i}}$ S. I. Jalal, ${ }^{\mathrm{j}}$ J. Fiore, ${ }^{\mathrm{k}}{ }^{*}$ S. Saraf, ${ }^{\mathrm{k}}$ H. Raftopoulos, ${ }^{\mathrm{k} \dagger}$ L. Gandhi ${ }^{1, \mathrm{~m}}$ :

${ }^{a}$ University of California San Francisco, Helen Diller Family Comprehensive Cancer Center, San Francisco, CA, USA; ' Massachusetts General Hospital Cancer Center and Harvard Medical School, Boston, MA, USA; 'Taussig Cancer Institute/Cleveland Clinic, Cleveland, OH, USA; ${ }^{\mathrm{d} S}$ Sanford Health, Sioux Falls, SD, USA; ' UPMC Hillman Cancer Center, Pittsburgh, PA, USA; ${ }^{f}$ University of Michigan, Ann Arbor, MI, USA; 'Hospital of the University of Pennsylvania,

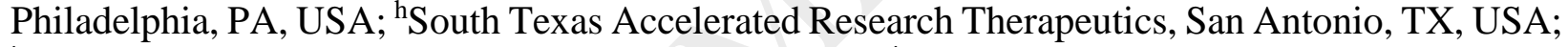
${ }^{\mathrm{i}}$ Fox Chase Cancer Center, Philadelphia, PA, USA; ' Indiana University School of Medicine, Indianapolis, IN, USA; ${ }^{k}$ Merck \& Co., Inc., Kenilworth, NJ, USA; 'Dana-Farber Cancer Institute, Boston, MA, USA; ${ }^{\mathrm{m} P e r l m u t t e r}$ Cancer Center - NYU Langone Medical Center, New York, NY, USA

*Currently at Bristol-Myers Squibb, Princeton, NJ, USA

${ }^{\dagger}$ Currently at Bayer US, LLC, Whippany, NJ, USA

Currently at Eli Lilly and Company, New York, NY, USA

"Corresponding author: Dr Matthew A. Gubens, MD, MS Associate Professor, Thoracic Medical Oncology University of California San Francisco, 1600 Divisadero St, San Francisco, CA 94143, Phone: (415) 885-3882, Email: matthew.gubens@ucsf.edu

This is the author's manuscript of the article published in final edited form as:

Gubens, M. A., Sequist, L. V., Stevenson, J. P., Powell, S. F., Villaruz, L. C., Gadgeel, S. M., ... Gandhi, L. (2018). Pembrolizumab in Combination with Ipilimumab as Second-Line or Later Therapy for Advanced Non-Small-Cell Lung Cancer: KEYNOTE-021 Cohorts D and H. Lung Cancer. https://doi.org/10.1016/j.lungcan.2018.12.015 
Target journal:

Word count:

Tables/figures:

References

\author{
Lung Cancer
}

3862 (limit, 5000 [includes main text only])

5 (limit, 6)

40 (limit, none specified)

\title{
Highlights
}

- KEYNOTE-021 evaluated pembrolizumab plus ipilimumab in second-line advanced NSCLC

- Antitumor activity was observed with pembrolizumab $2 \mathrm{mg} / \mathrm{kg}$ plus ipilimumab $1 \mathrm{mg} / \mathrm{kg}$

- Efficacy in these heavily pretreated patients was similar to historical controls

- This combination dosed every 3 weeks was associated with meaningful toxicity

\begin{abstract}
Objectives: Combination immunotherapy may result in improved antitumor activity compared with single-agent treatment. We report results from dose-finding and dose-expansion cohorts of the phase 1/2 KEYNOTE-021 study that evaluated combination therapy with anti-programmed death 1 (PD-1) antibody pembrolizumab plus anti-cytotoxic T-lymphocyte antigen-4 (CTLA-4) antibody ipilimumab in patients with previously treated advanced non-small-cell lung cancer (NSCLC).
\end{abstract}


Pembrolizumab Plus Ipilimumab as Second-Line Therapy for Advanced NSCLC

Materials and Methods: Eligibility criteria stipulated histologically/cytologically confirmed advanced NSCLC and treatment failure on $\geq 1$ prior systemic therapy (platinum-based chemotherapy or targeted therapy for patients with $E G F R / A L K$ aberrations). In the dose-finding cohort, patients initially received pembrolizumab $10 \mathrm{mg} / \mathrm{kg}$ plus ipilimumab 1 or $3 \mathrm{mg} / \mathrm{kg}$ once every 3 weeks for 4 cycles followed by pembrolizumab $10 \mathrm{mg} / \mathrm{kg}$ monotherapy for up to 2 years. Based on emerging published data, subsequent patients received pembrolizumab $2 \mathrm{mg} / \mathrm{kg}$ plus ipilimumab $1 \mathrm{mg} / \mathrm{kg}$. Objective response rate (ORR; primary efficacy endpoint) was assessed per RECIST version 1.1 by blinded, independent central review. Phase 2 hypothesis that ORR would be greater than the $20 \%$ rate for historical controls was evaluated using the exact binomial test.

Results: Fifty-one patients were enrolled; $71 \%$ received $\geq 2$ prior lines of therapy. No dose-limiting toxicities occurred at any dose level. Among patients who received pembrolizumab $2 \mathrm{mg} / \mathrm{kg}$ plus ipilimumab $1 \mathrm{mg} / \mathrm{kg}(\mathrm{n}=44)$, ORR was 30\% (95\% CI, 17\%-45\%), but not statistically significantly $>20 \%(P=0.0858)$. Median progression-free survival in this group was 4.1 (95\% CI, 1.4-5.8) months; median overall survival was 10.9 (95\% CI, 6.1-23.7) months. With pembrolizumab 2 $\mathrm{mg} / \mathrm{kg}$ plus ipilimumab $1 \mathrm{mg} / \mathrm{kg}$, incidences of treatment-related adverse events, grade 3-5 treatment-related adverse events, and immune-mediated adverse events and infusion reactions were $64 \%, 29 \%$ and $42 \%$, respectively.

Conclusions: In patients with heavily pretreated advanced NSCLC, pembrolizumab plus ipilimumab showed evidence of antitumor activity, but was associated with meaningful toxicity.

Keywords: Drug Therapy, Combination; Carcinoma, Non-Small-Cell Lung; pembrolizumab; ipilimumab 
Pembrolizumab Plus Ipilimumab as Second-Line Therapy for Advanced NSCLC

Abstract word count: 295 (limit, 300) 
Pembrolizumab Plus Ipilimumab as Second-Line Therapy for Advanced NSCLC

\section{Introduction}

Immunotherapy with checkpoint inhibitors has transformed the treatment of advanced non-smallcell lung cancer (NSCLC) [1]. Immune checkpoint inhibitors have been shown to improve outcomes, including overall survival (OS), compared with chemotherapy in both the first- [2] and second-line [3-7] settings when administered as monotherapy.

The humanized monoclonal antibody pembrolizumab blocks the interaction between programmed death 1 (PD-1) and its ligands programmed death ligand 1 and 2 (PD-L1, PD-L2) [8]. Binding of PD-1 on T cells by PD-L1 on tumor cells suppresses T cell effector functions; treatment with pembrolizumab results in reactivation of the antitumor immune response, as evidenced by an increase in intratumoral CD8-positive $\mathrm{T}$ cells [9]. In the phase 2/3 KEYNOTE-010 study, pembrolizumab significantly improved OS and objective response rate (ORR) compared with docetaxel in patients with previously treated advanced NSCLC with PD-L1 expression on $\geq 1 \%$ of tumor cells (tumor proportion score $[\mathrm{TPS}] \geq 1 \%$ ) [5]. Compared with standard platinum-based chemotherapy, pembrolizumab monotherapy improved progression-free survival (PFS) and OS in patients with previously untreated NSCLC of any histology with PD-L1 TPS $\geq 50 \%$ in the KEYNOTE-024 study [2] and improved OS in patients with PD-L1 TPS $\geq 1 \%$ in the KEYNOTE042 study [10]. In cohort G of the phase 2 KEYNOTE-021 study, pembrolizumab plus pemetrexed and carboplatin improved ORR and PFS compared with pemetrexed and carboplatin alone in patients with nonsquamous NSCLC, independent of PD-L1 status [11]. Recently, in the phase 3 KEYNOTE-189 study, pembrolizumab plus pemetrexed-platinum chemotherapy improved OS and PFS compared to placebo plus pemetrexed-platinum in patients with previously untreated metastatic nonsquamous NSCLC [12]. Similarly, in the phase 3 KEYNOTE-407 study, pembrolizumab plus carboplatin-paclitaxel/nab-paclitaxel improved OS and PFS compared with 
Pembrolizumab Plus Ipilimumab as Second-Line Therapy for Advanced NSCLC

placebo plus carboplatin-paclitaxel/nab-paclitaxel in patients with previously untreated metastatic squamous NSCLC [13].

Ipilimumab is a humanized monoclonal antibody against cytotoxic T-lymphocyte antigen-4 (CTLA-4), a molecule expressed on the surface of $\mathrm{T}$ cells that negatively regulates interleukin-2 production as well as T-cell proliferation and survival [14]. Treatment with ipilimumab has been associated with clonal expansion of CD8-positive $\mathrm{T}$ cells [15]. Ipilimumab has demonstrated activity in the treatment of melanoma [16,17], and as first-line therapy in advanced NSCLC when combined with anti-PD-1 therapy [18,19]. Notably, although results from a phase 2 study suggested a benefit with ipilimumab when used in a phased regimen with carboplatin-paclitaxel [20], no benefit was observed in a phase 3 trial evaluating this regimen [21].

Combining immunotherapies such as anti-PD-1 and anti-CTLA-4 agents, which target complementary pathways in the cancer-immunity cycle, might result in additive or synergistic antitumor activity compared with single-agent therapy [22,23]. In support of this hypothesis, the combination of nivolumab and ipilimumab has been shown to improve survival compared with ipilimumab alone in patients with previously untreated advanced NSCLC (only PFS data reported to date) [19] and melanoma albeit with an increased risk of grade 3 or 4 toxicity [24,25]. KEYNOTE-021 (ClinicalTrials.gov, NCT02039674) is a multicohort phase 1/2 study evaluating pembrolizumab-based combination therapies in patients with NSCLC. We report results for cohorts D (dose-finding cohort) and $\mathrm{H}$ (dose-expansion cohort), which evaluated pembrolizumab plus ipilimumab as second-line therapy for patients with advanced NSCLC. The primary objectives of these cohorts were to identify a recommended phase 2 dose for pembrolizumab in combination with ipilimumab and to evaluate antitumor activity of the combination. 
Pembrolizumab Plus Ipilimumab as Second-Line Therapy for Advanced NSCLC

\section{Methods}

\subsection{Eligibility}

The study populations for cohorts $\mathrm{D}$ and $\mathrm{H}$ of KEYNOTE-021 consisted of adults with histologically or cytologically confirmed stage IIIB/IV NSCLC (any histology) who had previously received $\geq 1$ platinum-based chemotherapy or an appropriate targeted therapy and had experienced treatment failure; multiple prior lines of treatment were permitted. Eligibility criteria also stipulated that patients have $\geq 1$ measurable lesion per Response Evaluation Criteria in Solid Tumors (RECIST) version 1.1 [26], Eastern Cooperative Oncology Group (ECOG) performance status of 0 to 1 , life expectancy $\geq 3$ months, adequate organ function, and provision of a tumor biopsy for assessment of PD-L1 expression. Key exclusion criteria included any previous treatment targeting immunoregulatory receptors or mechanisms (eg, PD-1, PD-L1, and CTLA-4), receipt of $>30$ Gy of radiation to the lungs in the previous 6 months, active autoimmune disease requiring systemic treatment (ie, disease-modifying agents, corticosteroids, immunosuppressive drugs) in the previous 2 years, active interstitial lung disease or history of pneumonitis requiring intravenous glucocorticoids, and untreated brain metastases (stable, treated metastases allowed).

Patients provided written informed consent. The study protocol was approved by an institutional review board at each site (protocol number MK-3475-021-03). The study was conducted in accordance with Good Clinical Practice guidelines and the Declaration of Helsinki.

\subsection{Study Design}

In both cohorts, patients received four 3-week cycles of pembrolizumab plus ipilimumab, with ipilimumab administered $\geq 30$ minutes after pembrolizumab infusion, followed by pembrolizumab monotherapy every three weeks (Q3W) for up to 2 years. Cohort D was a dose-finding cohort with 
Pembrolizumab Plus Ipilimumab as Second-Line Therapy for Advanced NSCLC

a 3+3 design in which dose-limiting toxicities (DLTs) during the first 3-week treatment cycle were evaluated in order to determine the recommended phase 2 dose of pembrolizumab in combination with ipilimumab. A DLT was defined as a treatment-related grade 3 nonhematologic, nonlaboratory toxicity lasting >3 days despite optimal supportive care; grade 4 nonhematologic, nonlaboratory toxicity; grade $3 / 4$ nonhematologic laboratory value persisting for $>1$ week or requiring medical intervention or hospitalization; grade 4 hematologic toxicity lasting $\geq 7$ days; grade $3 / 4$ febrile neutropenia; thrombocytopenia $<25,000 / \mathrm{mm}^{3}$ requiring elective platelet transfusion, urgent intervention, or intensive care unit admission; delay in initiating cycle 2 for $>2$ weeks due to treatment-related toxicity; or a grade 5 event.

The first 3 patients enrolled in cohort D received pembrolizumab $10 \mathrm{mg} / \mathrm{kg}$ plus ipilimumab 1 $\mathrm{mg} / \mathrm{kg}$. If $\leq 1$ DLT occurred during cycle 1,3 additional patients were to be enrolled at that dose level. If no DLTs occurred, the dose level was escalated and 3 patients were to be enrolled at a dose level of pembrolizumab $10 \mathrm{mg} / \mathrm{kg}$ plus ipilimumab $3 \mathrm{mg} / \mathrm{kg}$. If $\leq 1$ DLT occurred during cycle 1 at this dose level, 3 additional patients were to be enrolled.

Although no DLTs occurred among the first 6 patients enrolled in cohort D, safety concerns external to this trial (including treatment-related deaths) were noted in a phase 1 study evaluating nivolumab plus ipilimumab in patients with advanced NSCLC [18,27]. This led to a protocol amendment stipulating that all subsequent patients enrolled in cohort D receive pembrolizumab 2 $\mathrm{mg} / \mathrm{kg}$ plus ipilimumab $1 \mathrm{mg} / \mathrm{kg}$. If $\leq 1$ DLT occurred during cycle 1 at this lower dose, 3 additional patients were to be enrolled. If $\leq 1$ of the first 6 patients treated with pembrolizumab $2 \mathrm{mg} / \mathrm{kg}$ plus ipilimumab $1 \mathrm{mg} / \mathrm{kg}$ had DLTs, the dose was to be considered the recommended phase 2 dose and 
Pembrolizumab Plus Ipilimumab as Second-Line Therapy for Advanced NSCLC

6 additional patients would be enrolled to receive this dose. All patients in cohort $\mathrm{H}$ (dose expansion) received pembrolizumab $2 \mathrm{mg} / \mathrm{kg}$ plus ipilimumab $1 \mathrm{mg} / \mathrm{kg}$.

Study treatment continued until confirmed disease progression by RECIST version 1.1, intolerable toxicity, investigator decision, or withdrawal of consent. Clinically stable patients with radiologic evidence of disease progression could continue treatment until progression was confirmed $\geq 4$ weeks later. Treatment with pembrolizumab was withheld in the event of severe or life-threatening treatment-related toxicities. Ipilimumab dosing could be modified as clinically indicated, in accordance with the ipilimumab prescribing information.

The primary objective in part 1 (cohort D) was determination of a recommended phase 2 dose; evaluation of antitumor activity per RECIST version 1.1 was a secondary objective. The primary objective in part 2 (cohort $\mathrm{H})$ was evaluation of antitumor activity of the combination as assessed by the ORR per RECIST version 1.1; evaluation of PFS, OS, and duration of response were secondary objectives.

\subsection{Assessments}

Radiographic imaging was performed at baseline, every 6 weeks for the first 18 weeks, every 9 weeks during the remainder of the first year, and every 12 weeks during the second year. Response was assessed per RECIST version 1.1 by blinded, independent central review. Adverse events (AEs) and laboratory abnormalities were monitored during the study and graded per National Cancer Institute Common Terminology Criteria for Adverse Events (NCI CTCAE) version 4.0. At baseline, formalin-fixed tumor samples were evaluated for PD-L1 expression from core needle biopsies, excisional biopsies, or resected tissue collected at the time of diagnosis of metastatic 
Pembrolizumab Plus Ipilimumab as Second-Line Therapy for Advanced NSCLC

disease. Assessment was performed at a central laboratory using the PD-L1 IHC 22C3 pharmDx assay (Agilent Technologies, Carpinteria, CA). PD-L1 expression was reported as TPS.

\subsection{Statistical Analysis}

Analyses included all patients who received study treatment. Results from cohorts D and H for patients who received pembrolizumab $2 \mathrm{mg} / \mathrm{kg}$ plus ipilimumab $1 \mathrm{mg} / \mathrm{kg}$ were combined for analysis, as prespecified in the protocol. The difference between the observed ORR and the historical control rate of $20 \%$ was evaluated using a one-sided exact binomial test. With 44 patients, the study had approximately $90 \%$ power to detect a $20 \%$ difference between the observed and historical ORR at $\alpha=0.05$. PFS, OS, and duration of response were estimated using the KaplanMeier method. PFS was defined as the time from start of treatment to the earlier of disease progression or death; OS was determined based on the time from start of treatment to death; and duration of response was defined as the time from the first documented evidence of objective response to the earlier of disease progression or death. 
Pembrolizumab Plus Ipilimumab as Second-Line Therapy for Advanced NSCLC

\section{Results}

\subsection{Patients}

Fifty-one patients from 11 sites in the United States were enrolled in cohorts D and H. This included 18 patients in cohort D enrolled between February 27, 2014 and January 29, 2015 who received pembrolizumab $10 \mathrm{mg} / \mathrm{kg}$ plus ipilimumab $1 \mathrm{mg} / \mathrm{kg}$ (n=3), pembrolizumab $10 \mathrm{mg} / \mathrm{kg}$ plus ipilimumab $3 \mathrm{mg} / \mathrm{kg}(\mathrm{n}=3)$, or pembrolizumab $2 \mathrm{mg} / \mathrm{kg}$ plus ipilimumab $1 \mathrm{mg} / \mathrm{kg}(\mathrm{n}=12)$. The remaining 33 patients were enrolled in cohort H between April 6, 2015 and December 14, 2015 and received pembrolizumab $2 \mathrm{mg} / \mathrm{kg}$ plus ipilimumab $1 \mathrm{mg} / \mathrm{kg}$. Most patients had ECOG performance status of 1,22\% had an EGFR mutation, and $29 \%$ had brain metastases. The majority of patients ( $71 \%$ ) had received $\geq 2$ lines of prior therapy, and $33 \%$ had received $\geq 3$ prior therapies (Table 1). One patient in the pembrolizumab $2 \mathrm{mg} / \mathrm{kg}$ plus ipilimumab $1 \mathrm{mg} / \mathrm{kg}$ group had an original diagnosis of NSCLC that was later discovered to be metastatic melanoma; this patient was excluded from efficacy analyses but included in safety analyses. As of the cutoff date (November 7, 2016), 1 of 6 patients who received pembrolizumab $10 \mathrm{mg} / \mathrm{kg}$ plus ipilimumab $1 \mathrm{mg} / \mathrm{kg}$ completed 2 years of treatment; the remaining 5 patients had discontinued (progressive disease, $\mathrm{n}=4$; physician decision, $\mathrm{n}=1$ ). For these 6 patients, median follow-up duration was 20.8 (range, 6.5-31.6) months, and median number of cycles of pembrolizumab was 9.5 (range, 3-35). Among patients who received pembrolizumab $2 \mathrm{mg} / \mathrm{kg}$ plus ipilimumab $1 \mathrm{mg} / \mathrm{kg}(\mathrm{n}=45), 1$ patient completed treatment, 2 were ongoing, and 42 had discontinued (disease progression, $n=22 ; \mathrm{AE}$, $\mathrm{n}=10$; clinical progression, $\mathrm{n}=8$; withdrawal of consent, $\mathrm{n}=2$ ). Median follow-up duration for these patients was 11.3 (range, 0.3-26.9) months, and they received a median of 4 (range, 1-35) cycles of pembrolizumab. 
Pembrolizumab Plus Ipilimumab as Second-Line Therapy for Advanced NSCLC

\subsection{Dose-Limiting Toxicities}

No DLTs occurred among patients in either of the 2 pembrolizumab $10 \mathrm{mg} / \mathrm{kg}$ ipilimumab dose groups. Similarly, no DLTs occurred among the 12 patients who were enrolled after the protocol amendment and received pembrolizumab $2 \mathrm{mg} / \mathrm{kg}$ plus ipilimumab $1 \mathrm{mg} / \mathrm{kg}$. Therefore, the requirements for dose expansion were met.

\subsection{Efficacy}

Among the 6 patients who received pembrolizumab $10 \mathrm{mg} / \mathrm{kg}, 3$ had partial responses, yielding an ORR of 50\% (Table 2). Median time to response was 1.4 (range, 1.4-20.7) months, and median duration of response had not been reached (range, 8.3 [ongoing] to 28.8 [ongoing]). At the time of analysis, 3 patients had died.

Among patients who received pembrolizumab $2 \mathrm{mg} / \mathrm{kg}$ plus ipilimumab $1 \mathrm{mg} / \mathrm{kg}$ and were included in the efficacy analyses $(n=44), 13$ had an objective response ( 1 complete response, 12 partial responses), and 11 had stable disease as their best response. The ORR was 30\% (95\% CI, $17 \%-45 \%$ ), which was not significantly greater than the $20 \%$ threshold specified in the primary hypothesis $(P=0.0858)$. The disease control rate (complete response + partial response + stable disease) was 55\%. Among patients with a response, median (range) time to response was 2.7 (1.36.2) months, and median duration of response was 10.5 (2.5 [ongoing] to 21.0) months. ORR was $25 \%(5 / 20)$ in patients with PD-L1 TPS <1\%, 39\% (7/18) among patients with TPS $1 \%$ to $49 \%$, and $17 \%$ (1/6) in patients with TPS $\geq 50 \%$. Of 10 patients with $E G F R$ mutations, 1 had an objective response (ORR, 10\%). ORRs were 38\% (5/13) in patients with <2 lines of prior therapy and $26 \%$ $(8 / 31)$ in those with $\geq 2$ lines of prior therapy. Best response change from baseline in the sum of longest diameters of target lesions is shown in Figure 1. 
Pembrolizumab Plus Ipilimumab as Second-Line Therapy for Advanced NSCLC

At the time of analysis, 39 patients had died or experienced disease progression; median PFS was 4.1 (95\% CI, 1.4-5.8) months, and the 6-month PFS rate was 32\% (95\% CI, 19\%-46\%; Figure 2A). Twenty-six patients had died; median OS was 10.9 (95\% CI, 6.1-23.7) months with a 6month OS rate of $67 \%$ (95\% CI, 51\%-79\%; Figure 2B).

\subsection{Toxicity}

Among the 6 patients who received pembrolizumab $10 \mathrm{mg} / \mathrm{kg}, 1$ patient who received pembrolizumab $10 \mathrm{mg} / \mathrm{kg}$ plus ipilimumab $1 \mathrm{mg} / \mathrm{kg}$ had a treatment-related grade 3 reduction in lymphocyte count; there were no other grade 3-5 treatment-related AEs among these patients (Table 3). Immune-mediated AEs and infusion reactions (regardless of attribution to study treatment or immune relatedness by the investigator) occurred in 2 patients: one patient had grade 2 hypothyroidism, and a second patient had grade 1 pneumonitis and uveitis (grade 2).

Among the 45 patients who received pembrolizumab $2 \mathrm{mg} / \mathrm{kg}$ plus ipilimumab $1 \mathrm{mg} / \mathrm{kg}, 44$ (98\%) experienced $\geq 1$ AE, irrespective of relationship to study treatment, with 22 (49\%) having grade 3 5 AEs. Treatment-related AEs occurred in 29/45 patients (64\%), with fatigue, hypothyroidism, decreased appetite, diarrhea, and pruritus each occurring in $>10 \%$ of patients (Table 3). Grade $3-$ 5 treatment-related AEs occurred in 13/45 patients (29\%), with colitis being the only event to occur in more than one patient $(n=2$; both grade 3$)$. Three patients had grade 4 treatment-related AEs (pancytopenia, large-intestine perforation, and diabetic ketoacidosis), and there was 1 treatmentrelated death (pancreatitis occurring during cycle 23). Treatment-related AEs led to discontinuation of pembrolizumab and/or ipilimumab in $9(20 \%)$ patients (pancytopenia, hyperthyroidism, hypothyroidism, colitis, large intestine perforation, pancreatitis, fatigue, alanine aminotransferase increased, aspartate aminotransferase increased, diabetic ketoacidosis, and 
Pembrolizumab Plus Ipilimumab as Second-Line Therapy for Advanced NSCLC

myasthenia gravis); no individual event resulted in treatment discontinuation in $>1$ patient. Immune-mediated AEs and infusion reactions occurred in 19 (42\%) patients; hypothyroidism was the only immune-mediated event reported in $>10 \%$ of patients (Table 3). Eight (18\%) patients had grade 3-5 immune-mediated AEs and infusion reactions: grade 5 pancreatitis $(n=1)$; grade 4 diabetic ketoacidosis $(n=1)$; and grade 3 severe skin reactions $(n=3)$, colitis $(n=2)$, adrenal insufficiency $(\mathrm{n}=1)$, and pneumonitis $(\mathrm{n}=1)$. 
Pembrolizumab Plus Ipilimumab as Second-Line Therapy for Advanced NSCLC

\section{Discussion}

In this phase $1 / 2$ study evaluating pembrolizumab plus ipilimumab in patients with previously treated NSCLC, more than one-third of whom had received $\geq 3$ lines of prior therapy, we found evidence of antitumor activity, but the ORR of $30 \%$ with pembrolizumab $2 \mathrm{mg} / \mathrm{kg}$ plus ipilimumab $1 \mathrm{mg} / \mathrm{kg}$ did not meet the prespecified endpoint of being significantly greater than $20 \%$ (ie, superiority over historical control; $P=0.0858$ ). Although no DLTs occurred at any of the dose levels tested, the combination of pembrolizumab and ipilimumab was associated with meaningful toxicity in patients with previously treated advanced NSCLC .

Safety data external to the current study - including 3 treatment-related deaths among 49 patients in an interim analysis of several of the early dose cohorts in the CheckMate 012 study, which evaluated nivolumab plus ipilimumab in advanced NSCLC $[18,27]$ —led to a decision to evaluate the efficacy of pembrolizumab plus ipilimumab at doses of pembrolizumab $2 \mathrm{mg} / \mathrm{kg}$ and ipilimumab $1 \mathrm{mg} / \mathrm{kg}$. At this dose level, the combination of pembrolizumab plus ipilimumab was associated with meaningful toxicity that was greater than that observed with pembrolizumab monotherapy in previously treated advanced NSCLC [5]. In the current study, 20\% discontinued treatment due to a treatment-related $\mathrm{AE}$ and $29 \%$ experienced 1 or more treatment-related grade 3-5 AEs, including 3 who experienced grade 4 events and 1 patient who died. In the KEYNOTE010 study, which evaluated pembrolizumab monotherapy in previously treated advanced NSCLC, percentages of patients who discontinued due to treatment-related AEs (4\% and 5\% with pembrolizumab $2 \mathrm{mg} / \mathrm{kg}$ and $10 \mathrm{mg} / \mathrm{kg}$, respectively) and percentages with treatment-related grade 3-5 AEs (13\% and 16\%, respectively) were comparatively lower [5]. Whereas, in the KEYNOTE024 study, which evaluated pembrolizumab monotherapy in treatment-naive patients with advanced NSCLC and PD-L1 TPS $\geq 50 \%$, the rate of treatment-related AEs was higher compared 
Pembrolizumab Plus Ipilimumab as Second-Line Therapy for Advanced NSCLC

to the current study $(73 \%$ vs $64 \%$ in patients who received pembrolizumab $2 \mathrm{mg} / \mathrm{kg}$ plus ipilimumab $1 \mathrm{mg} / \mathrm{kg}$ ), but the rate of discontinuation from those events was lower (7\% vs 20\%) [2]. In addition, in patients with melanoma, nivolumab plus ipilimumab combination regimens have demonstrated increased rates of AEs [24,25], some of which can be difficult to diagnose and manage [28,29]. It is possible that the high rate of discontinuation in our study may be due to similar challenges with immune-mediated AEs, and that increased experience with such combination regimens may result in improved strategies for toxicity management. Additionally, ipilimumab was dosed Q3W in our study, a dosing schedule that was not found suitable for further clinical development in the CheckMate 012 study [18]. The nature of observed treatment-related AEs in the current study (fatigue, hypothyroidism, decreased appetite, diarrhea, and pruritus occurred most frequently) matched what would be anticipated given the immune mechanism of action of both agents, and the safety profile was generally consistent with that reported in studies evaluating nivolumab plus ipilimumab in advanced NSCLC [18,19] and melanoma [24,30].

Although the prespecified threshold for clinical improvement was not met, the ORR of $30 \%$ in this heavily pretreated group of patients, the majority of whom had received $\geq 2$ lines of prior therapy, compares favorably with the $18 \%$ rate reported for pembrolizumab $2 \mathrm{mg} / \mathrm{kg}$ monotherapy in the KEYNOTE-010 study, which enrolled previously treated patients with PD-L1 TPS $\geq 1 \%$, most of whom had received 1 prior line of therapy for advanced disease [5]. In addition, a higher proportion of patients had brain metastases and were positive for EGFR mutation at baseline in the current study compared with KEYNOTE-010 [5] and there were only 4 patients with squamous NSCLC enrolled, both of which could have potentially influenced the study outcome. PD-(L)1 inhibition has been shown to lead to similar or worse outcomes compared to chemotherapy in patients with EGFR mutations [31-33]. The limited number of patients with squamous histology in this study 
Pembrolizumab Plus Ipilimumab as Second-Line Therapy for Advanced NSCLC

precludes histology-based assessment of outcomes with this combination. In CheckMate 227, improvements in PFS were observed with the combination of nivolumab plus ipilimumab versus chemotherapy in treatment-naive patients with $\mathrm{TMB} \geq 10$ mutations/megabase with either squamous (HR, 0.63; 95\% CI, 0.39-1.04) or nonsquamous NSCLC (HR, 0.55; 95\% CI; 0.38-0.80)

[19]. However, these data are not conclusive, given that the $95 \%$ confidence intervals for the HR for squamous histology contained 1.

Notably, almost $50 \%$ of patients in the current study who were evaluable for efficacy at the pembrolizumab $2 \mathrm{mg} / \mathrm{kg}$ plus ipilimumab $1 \mathrm{mg} / \mathrm{kg}$ dose level had PD-L1 TPS <1\%, which has previously been shown to be associated with lower ORR with pembrolizumab monotherapy in the KEYNOTE-001 study [34]. Although there was little evidence of an influence of PD-L1 expression on the ORR in the current study, interpretation of this finding is limited by the relatively small number of patients enrolled. Nonetheless, with median follow-up of 11.3 months, median PFS and OS were 4.1 and 10.9 months, respectively. These outcomes were similar to those reported with pembrolizumab $2 \mathrm{mg} / \mathrm{kg}$ monotherapy after a similar follow-up duration in previously treated patients with PD-L1-positive advanced NSCLC in the KEYNOTE-010 study (PFS, 3.9 months; OS, 10.4 months, respectively) [5].

Other studies have evaluated anti-PD-1/anti-PD-L1 plus anti-CTLA-4 combinations in patients with advanced NSCLC. Though unacceptable toxicity was observed in earlier cohorts in the openlabel, phase 1, multicohort study CheckMate 012, which evaluated nivolumab plus ipilimumab in previously untreated advanced NSCLC, these cohorts received ipilimumab dosing Q $3 \mathrm{~W}$. With the combination of nivolumab $3 \mathrm{mg} / \mathrm{kg}$ Q2W plus ipilimumab $1 \mathrm{mg} / \mathrm{kg}$ at dosing intervals of Q6W or Q12W, manageable toxicity was observed after a median follow-up of approximately 1 year [18]. 
Pembrolizumab Plus Ipilimumab as Second-Line Therapy for Advanced NSCLC

When pooled across both dose schedules, ORR appeared to be greater with nivolumab plus ipilimumab than with nivolumab monotherapy (43\% vs $23 \%$ ); albeit this trial was not randomized across the combination and monotherapy cohorts. In contrast to findings from our study, there appeared to be an association between PD-L1 expression and ORR in CheckMate 012 (PD-L1 $<1 \%, 18 \%$; PD-L1 $\geq 1 \%, 57 \%$ ), although it is noteworthy that the PD-L1 assay and antibody used to determine TPS were different than those used in our study [18].

More recently, the open-label, multipart, phase 3 CheckMate 227 trial compared outcomes in treatment-naive patients with advanced NSCLC who received nivolumab $3 \mathrm{mg} / \mathrm{kg}$ Q2W plus ipilimumab $1 \mathrm{mg} / \mathrm{kg}$ Q6W compared with platinum doublet chemotherapy [19]. In part 1 of this study, the coprimary endpoint of median (95\% CI) PFS among patients with high tumor mutational burden $(\mathrm{TMB} ; \geq 10$ mutations/megabase) was longer in those who received nivolumab plus ipilimumab (7.2 months [5.5-13.2]) versus chemotherapy (5.5 months [4.4-5.8]) with a hazard ratio (HR) of $0.58(97.5 \% \mathrm{CI}, 0.41-0.81 ; P<0.001)$. However, similar results were not observed with nivolumab monotherapy in patients with $\mathrm{TMB} \geq 13$ mutations/megabase and PD-L1 $\geq 1 \%$ (HR for nivolumab monotherapy versus chemotherapy, $0.95 ; 97.5 \% \mathrm{CI}, 0.61-1.48 ; P=0.78)$. Of note, TMB was not assessed among our patients. In the overall population, irrespective of TMB or PDL1 expression, median PFS was 4.9 (95\% CI, 4.1-5.6) months with nivolumab plus ipilimumab, which was similar to the results from our current study, versus 5.5 (95\% CI, 4.6-5.6) months with chemotherapy [19].

In a phase $1 \mathrm{~b}$ dose-escalation study in patients with previously untreated NSCLC, the combination of the anti-PD-L1 antibody durvalumab and the anti-CTLA-4 antibody tremelimumab had a manageable tolerability profile and was associated with antitumor activity [35]. The maximum 
Pembrolizumab Plus Ipilimumab as Second-Line Therapy for Advanced NSCLC

tolerated dose was durvalumab $15 \mathrm{mg} / \mathrm{kg}$ Q4W plus tremelimumab $3 \mathrm{mg} / \mathrm{kg}$ once Q4W administered for 6 weeks, followed by once Q12W. Notably, responses occurred irrespective of tumor PD-L1 expression. It has recently been reported that in the MYSTIC trial, which compares this combination with platinum-based chemotherapy in patients with previously untreated NSCLC [36], the coprimary endpoint of improved PFS in patients with PD-L1 TPS $\geq 25 \%$ was not met; evaluation of the coprimary endpoint of OS is ongoing [37].

In conclusion, the combination of pembrolizumab plus ipilimumab had antitumor activity in heavily pretreated patients but was associated with meaningful toxicity. At present, pembrolizumab $200 \mathrm{mg}$ Q3W plus ipilimumab $1 \mathrm{mg} / \mathrm{kg}$ once Q6W is being evaluated in treatmentnaive patients with advanced NSCLC with PD-L1 TPS $\geq 50 \%$ in the randomized, placebocontrolled phase 3 KEYNOTE-598 study (ClinicalTrials.gov, NCT03302234), which was designed based on the experience from our current study [38]. KEYNOTE-598 will evaluate a different dosing schedule (ipilimumab Q6W rather than Q3W) specifically in patients with PD-L1 TPS $\geq 50 \%$ [38]. Additionally, ongoing phase 3 trials are evaluating nivolumab plus ipilimumab [39] and durvalumab plus tremelimumab [40] in patients with previously untreated NSCLC. These studies will provide further information on the efficacy and safety of anti-PD-1/anti-PD-L1 plus anti-CTLA-4 therapy in patients with advanced NSCLC. 
Pembrolizumab Plus Ipilimumab as Second-Line Therapy for Advanced NSCLC

\section{Acknowledgments}

The authors thank the patients and their families and all investigators and site personnel who participated in this study. Medical writing assistance was provided by Michael S. McNamara, MS, and Sheri Arndt, PharmD, of C4 MedSolutions, LLC (Yardley, PA), a CHC Group company. This assistance was funded by Merck Sharp \& Dohme Corp., a subsidiary of Merck \& Co., Inc., Kenilworth, NJ, USA.

\section{Funding}

Funding for this research was provided by Merck Sharp \& Dohme Corp., a subsidiary of Merck \& Co., Inc., Kenilworth, NJ, USA. The sponsor contributed to the study design; collection, analysis, and interpretation of data; and the writing of the report.

\section{Disclosures}

M. A. Gubens has served as an advisory board member for Abbvie, ARIAD, AstraZeneca, BristolMyers Squibb, Genentech/Roche, Mersana, and Novartis, and has received research funding from Celgene, Merck, Novartis, OncoMed, and Roche; L. V. Sequist has served as an advisory board member for Bristol-Myers Squibb, AstraZeneca, Pfizer, and Genentech, and as a consultant for Boehringer Ingelheim, Merrimack, Novartis, and Clovis Oncology; J. P. Stevenson has received research grant support provided to his institution by Bristol-Myers Squibb, Bayer Healthcare, Aduro Biotech, and Merck; S. F. Powell has received research funding from Merck, Bristol-Myers Squibb, Incyte, Genentech, Novartis, and Pfizer; L. C. Villaruz has no conflicts of interest to disclose; S. M. Gadgeel has received honoraria from Genentech/Roche and AstraZeneca, and has served in a consulting or advisory role for Pfizer, AstraZeneca, Genentech/Roche, ARIAD, Bristol-Myers Squibb, and Novartis; C. J. Langer has received personal fees for serving on a data 
Pembrolizumab Plus Ipilimumab as Second-Line Therapy for Advanced NSCLC

and safety monitoring committee for Eli Lilly, Synta, AbbVie, and Amgen, and for serving as an advisory board member for AstraZeneca, Clovis Oncology, Bristol-Myers Squibb, and Genetech/Roche, and has received research grant support provided to his institution by Merck, Clovis Oncology, GlaxoSmithKline, Genentech/Roche, Advantagene, and Inovio; A. Patnaik has received research funding provided to her institution by Merck; H. Borghaei has received clinical trial grant support from Merck, Bristol-Myers Squibb, Eli Lilly, and Celgene, and personal fees for serving in a consulting and/or advisory role for Merck, Bristol-Myers Squibb, Eli Lilly, Celgene, Genmab, Novartis, AstraZeneca, Pfizer, EMD Serono, and Boehringer Ingelheim; S. I. Jalal has received research funding from AstraZeneca; J. Fiore has received payment from BMS and Merck, was an employee of Merck Sharp \& Dohme Corp., a subsidiary of Merck \& Co., Inc., Kenilworth, NJ, USA until 26 January 2018, and is a current employee of Bristol-Myers Squibb; S. Saraf is a current employee of Merck Sharp \& Dohme Corp., a subsidiary of Merck \& Co., Inc., Kenilworth, NJ, USA; H. Raftopoulos was an employee of Merck Sharp \& Dohme Corp., a subsidiary of Merck \& Co., Inc., Kenilworth, NJ, USA until 19 September 2017, and is a current employee of Bayer US, LLC; L. Gandhi has served as an advisory board member for Genentech/Roche, Merck, AstraZeneca, and Ignyta, has served as a paid consultant for Celldex and Eli Lilly, has received research funding from the Bristol-Myers Squibb IION Foundation and Merck, and is a current employee of Eli Lilly and Company as of June 25, 2018. 
Pembrolizumab Plus Ipilimumab as Second-Line Therapy for Advanced NSCLC

\section{References}

1. Somasundaram A, Burns TF. The next generation of immunotherapy: keeping lung cancer in check. J Hematol Oncol 2017;10:87.

2. Reck M, Rodriguez-Abreu D, Robinson AG, Hui R, Csoszi T, Fulop A, et al. Pembrolizumab versus chemotherapy for PD-L1-positive non-small-cell lung cancer. N Engl J Med 2016;375:1823-33.

3. Brahmer J, Reckamp KL, Baas P, Crino L, Eberhardt WE, Poddubskaya E, et al. Nivolumab versus docetaxel in advanced squamous-cell non-small-cell lung cancer. N Engl J Med 2015;373:123-35.

4. Borghaei H, Paz-Ares L, Horn L, Spigel DR, Steins M, Ready NE, et al. Nivolumab versus docetaxel in advanced nonsquamous non-small-cell lung cancer. $\mathrm{N}$ Engl $\mathrm{J}$ Med $2015 ; 373: 1627-39$.

5. Herbst RS, Baas P, Kim DW, Felip E, Perez-Gracia JL, Han JY, et al. Pembrolizumab versus docetaxel for previously treated, PD-L1-positive, advanced non-small-cell lung cancer (KEYNOTE-010): a randomised controlled trial. Lancet 2016;387:1540-50.

6. Fehrenbacher L, Spira A, Ballinger M, Kowanetz M, Vansteenkiste J, Mazieres J, et al. Atezolizumab versus docetaxel for patients with previously treated non-small-cell lung cancer (POPLAR): a multicentre, open-label, phase 2 randomised controlled trial. Lancet 2016;387:1837-46.

7. Rittmeyer A, Barlesi F, Waterkamp D, Park K, Ciardiello F, von Pawel J, et al. Atezolizumab versus docetaxel in patients with previously treated non-small-cell lung cancer (OAK): a phase 3, open-label, multicentre randomised controlled trial. Lancet 2017;389:255-65. 
Pembrolizumab Plus Ipilimumab as Second-Line Therapy for Advanced NSCLC

8. Sul J, Blumenthal GM, Jiang X, He K, Keegan P, Pazdur R. FDA approval summary: pembrolizumab for the treatment of patients with metastatic non-small cell lung cancer whose tumors express programmed death-ligand 1. Oncologist 2016;21:643-50.

9. Tumeh PC, Harview CL, Yearley JH, Shintaku IP, Taylor EJ, Robert L, et al. PD-1 blockade induces responses by inhibiting adaptive immune resistance. Nature 2014;515:568-71.

10. Mok TSK, Wu Y-L, Kudaba I, Kowalski DM, Cho BC, Turna HZ, et al. Pembrolizumab versus chemotherapy for previously untreated, PD-L1-expressing, locally advanced or metastatic non-small-cell lung cancer (KEYNOTE-042): a randomised, open-label, controlled, phase 3 trial. Lancet:In press.

11. Langer CJ, Gadgeel SM, Borghaei H, Papadimitrakopoulou VA, Patnaik A, Powell SF, et al. Carboplatin and pemetrexed with or without pembrolizumab for advanced, nonsquamous non-small-cell lung cancer: a randomised, phase 2 cohort of the open-label KEYNOTE-021 study. Lancet Oncol 2016;17:1497-508.

12. Gandhi L, Rodriguez-Abreu D, Gadgeel S, Esteban E, Felip E, De Angelis F, et al. Pembrolizumab plus chemotherapy in metastatic non-small-cell lung cancer. N Engl J Med 2018;378:2078-92.

13. Paz-Ares L, Luft A, Vicente D, Tafreshi A, Gümüş M, Mazières J, et al. Pembrolizumab plus chemotherapy for squamous non-small-cell lung cancer. N Engl J Med 2018:DOI: 10.1056/NEJMoa1810865.

14. Buchbinder EI, Desai A. CTLA-4 and PD-1 pathways: similarities, differences, and implications of their inhibition. Am J Clin Oncol 2016;39:98-106. 
Pembrolizumab Plus Ipilimumab as Second-Line Therapy for Advanced NSCLC

15. Subudhi SK, Aparicio A, Gao J, Zurita AJ, Araujo JC, Logothetis CJ, et al. Clonal expansion of CD8 T cells in the systemic circulation precedes development of ipilimumabinduced toxicities. Proc Natl Acad Sci U S A 2016;113:11919-24.

16. Hodi FS, O'Day SJ, McDermott DF, Weber RW, Sosman JA, Haanen JB, et al. Improved survival with ipilimumab in patients with metastatic melanoma. $\mathrm{N}$ Engl $\mathrm{J}$ Med 2010;363:711-23.

17. Eggermont AM, Chiarion-Sileni V, Grob JJ, Dummer R, Wolchok JD, Schmidt H, et al. Adjuvant ipilimumab versus placebo after complete resection of high-risk stage III melanoma (EORTC 18071): a randomised, double-blind, phase 3 trial. Lancet Oncol 2015;16:522-30.

18. Hellmann MD, Rizvi NA, Goldman JW, Gettinger SN, Borghaei H, Brahmer JR, et al. Nivolumab plus ipilimumab as first-line treatment for advanced non-small-cell lung cancer (CheckMate 012): results of an open-label, phase 1, multicohort study. Lancet Oncol 2017;18:31-41.

19. Hellmann MD, Ciuleanu TE, Pluzanski A, Lee JS, Otterson GA, Audigier-Valette C, et al. Nivolumab plus ipilimumab in lung cancer with a high tumor mutational burden. N Engl J Med 2018;378:2093-104.

20. Lynch TJ, Bondarenko I, Luft A, Serwatowski P, Barlesi F, Chacko R, et al. Ipilimumab in combination with paclitaxel and carboplatin as first-line treatment in stage IIIB/IV nonsmall-cell lung cancer: results from a randomized, double-blind, multicenter phase II study. J Clin Oncol 2012;30:2046-54.

21. Randomized, Multicenter, Double-Blind, Phase 3 Trial Comparing the Efficacy of Ipilimumab in Addition to Paclitaxel and Carboplatin Versus Placebo in Addition to 
Pembrolizumab Plus Ipilimumab as Second-Line Therapy for Advanced NSCLC

Paclitaxel and Carboplatin in Subjects With Stage IV/Recurrent Non Small Cell Lung $\begin{array}{llll}\text { Cancer } & \text { (NSCLC). } & \text { Available }\end{array}$ https:/clinicaltrials.gov/ct2/show/results/NCT01285609?term=carboplatin\&intr=ipilimu mab\&phase=2\&rank=2\&sect=X01256\#all Accessed September 22, 2017.

22. Heist RS. PD-(L)1 inhibitors and CTLA-4 inhibitors: rationale for combinations and recent data in non-small cell lung cancer. Am J Hematol Oncol 2015;11:21-5.

23. Ott PA, Hodi FS, Kaufman HL, Wigginton JM, Wolchok JD. Combination immunotherapy: a road map. J Immunother Cancer 2017;5:16.

24. Larkin J, Chiarion-Sileni V, Gonzalez R, Grob JJ, Cowey CL, Lao CD, et al. Combined nivolumab and ipilimumab or monotherapy in untreated melanoma. $\mathrm{N}$ Engl $\mathrm{J}$ Med 2015;373:23-34.

25. Larkin J, Chiarion-Sileni V, Gonzalez R, Rutkowski P, Grob JJ, Cowey CL, et al. Overall survival (OS) results from a phase III trial of nivolumab (NIVO) combined with ipilimumab (IPI) in treatment-naïve patients with advanced melanoma (CheckMate 067). Cancer Res 2017;77.

26. Eisenhauer EA, Therasse P, Bogaerts J, Schwartz LH, Sargent D, Ford R, et al. New response evaluation criteria in solid tumours: revised RECIST guideline (version 1.1). Eur J Cancer 2009;45:228-47.

27. Antonia SJ, Gettinger S, Goldman J, Chow LQ, Juergens R, Borghaei H, et al. Safety and efficacy of first-line nivolumab (anti-PD-1; BMS-936558; ONO-4538) and ipilimumab in non-small cell lung cancer (NSCLC). Int J Radiat Oncol Biol Phys 2014;90:S32-S3.

28. Gerson JN, Ramamurthy C, Borghaei H. Managing adverse effects of immunotherapy. Clin Adv Hematol Oncol 2018;16:364-74. 
Pembrolizumab Plus Ipilimumab as Second-Line Therapy for Advanced NSCLC

29. Larkin J, Chmielowski B, Lao CD, Hodi FS, Sharfman W, Weber J, et al. Neurologic Serious Adverse Events Associated with Nivolumab Plus Ipilimumab or Nivolumab Alone in Advanced Melanoma, Including a Case Series of Encephalitis. Oncologist 2017;22:70918.

30. Postow MA, Chesney J, Pavlick AC, Robert C, Grossmann K, McDermott D, et al. Nivolumab and ipilimumab versus ipilimumab in untreated melanoma. N Engl J Med 2015;372:2006-17.

31. Lee CK, Man J, Lord S, Links M, Gebski V, Mok T, et al. Checkpoint inhibitors in metastatic EGFR-mutated non-small cell lung cancer--a meta-analysis. J Thorac Oncol 2017;12:403-7.

32. Dong ZY, Zhang JT, Liu SY, Su J, Zhang C, Xie Z, et al. EGFR mutation correlates with uninflamed phenotype and weak immunogenicity, causing impaired response to PD-1 blockade in non-small cell lung cancer. Oncoimmunology 2017;6:e1356145.

33. Soo RA, Lim SM, Syn NL, Teng R, Soong R, Mok TSK, et al. Immune checkpoint inhibitors in epidermal growth factor receptor mutant non-small cell lung cancer: Current controversies and future directions. Lung Cancer 2018;115:12-20.

34. Garon EB, Rizvi NA, Hui R, Leighl N, Balmanoukian AS, Eder JP, et al. Pembrolizumab for the treatment of non-small-cell lung cancer. N Engl J Med 2015;372:2018-28.

35. Antonia S, Goldberg SB, Balmanoukian A, Chaft JE, Sanborn RE, Gupta A, et al. Safety and antitumour activity of durvalumab plus tremelimumab in non-small cell lung cancer: a multicentre, phase 1b study. Lancet Oncol 2016;17:299-308.

36. A Phase III Randomized, Open-Label, Multi-Center, Global Study of MEDI4736 in Combination With Tremelimumab Therapy or MEDI4736 Monotherapy Versus Standard 
Pembrolizumab Plus Ipilimumab as Second-Line Therapy for Advanced NSCLC

of Care Platinum-Based Chemotherapy in First Line Treatment of Patients With Advanced or Metastatic Non Small-Cell Lung Cancer (NSCLC) (MYSTIC). Available at: https://clinicaltrials.gov/show/NCT02453282. Accessed September 26, 2017.

37. AstraZeneca reports initial results from the ongoing MYSTIC trial in Stage IV lung cancer. Available at: $\quad$ https://www.astrazeneca-us.com/content/az-us/media/pressreleases/2017/astrazeneca-reports-initial-results-from-the-ongoing-mystic-trial-in-stageiv-lung-cancer-07272017.html. Accessed September 26, 2017.

38. Boyer M, McLean J, Xu L, Samkari A, Carbone D. Pembrolizumab Plus Ipilimumab or Placebo in 1L Metastatic NSCLC with PD-L1 Tumor Proportion Score (TPS) $\geq 50 \%$ : KEYNOTE-598. J Thorac Oncol 2018;13:S462.

39. Hellman M, Ramalingam S, Reck M, O'Byrne K, Paz-Ares L, Harbison CT, et al. An open label randomized phase III trial of nivolumab or nivolumab plus ipilimumab vs platinum doublet chemotherapy (PT-DC) in patients with chemotherapy-naïve stage IV or recurrent non-small cell lung cancer (NSCLC) (CheckMate 227). J Immunother Cancer 2015;3:P154.

40. Peters S, Antonia S, Goldberg SB, Heymach JV, Kim ES, Nakagawa K, et al. 191TiP: MYSTIC: a global, phase 3 study of durvalumab (MEDI4736) plus tremelimumab combination therapy or durvalumab monotherapy versus platinum-based chemotherapy (CT) in the first-line treatment of patients (pts) with advanced stage IV NSCLC. J Thorac Oncol 2016;11:S139-40. 
Pembrolizumab Plus Ipilimumab as Second-Line Therapy for Advanced NSCLC

\section{Figure Legends}

Figure 1. Change from baseline in the sum of longest diameters of target lesions in patients who received pembrolizumab $2 \mathrm{mg} / \mathrm{kg}$ plus ipilimumab $1 \mathrm{mg} / \mathrm{kg}$ in cohorts $\mathrm{D}$ and H. One patient who had an original diagnosis of NSCLC but was later determined to have metastatic melanoma was excluded from efficacy analyses but included in safety analyses; 12 additional patients are excluded from this figure because of missing target lesion information. *Patients with PD-L1 TPS $\geq 1 \%$. PD-L1 = programmed death ligand 1 ; TPS = tumor proportion score .

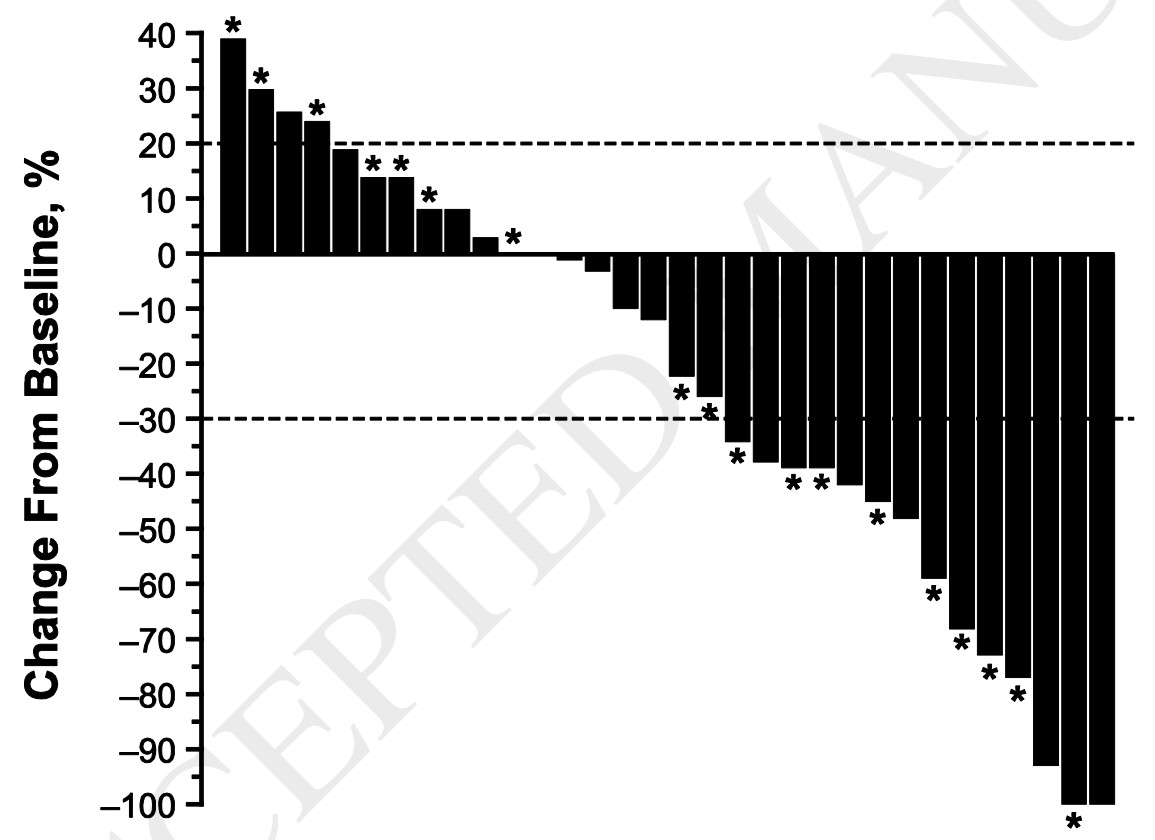


Pembrolizumab Plus Ipilimumab as Second-Line Therapy for Advanced NSCLC

Figure 2. Kaplan-Meier analysis of (A) progression-free survival and (B) overall survival among patients who received pembrolizumab $2 \mathrm{mg} / \mathrm{kg}$ plus ipilimumab $1 \mathrm{mg} / \mathrm{kg}$.
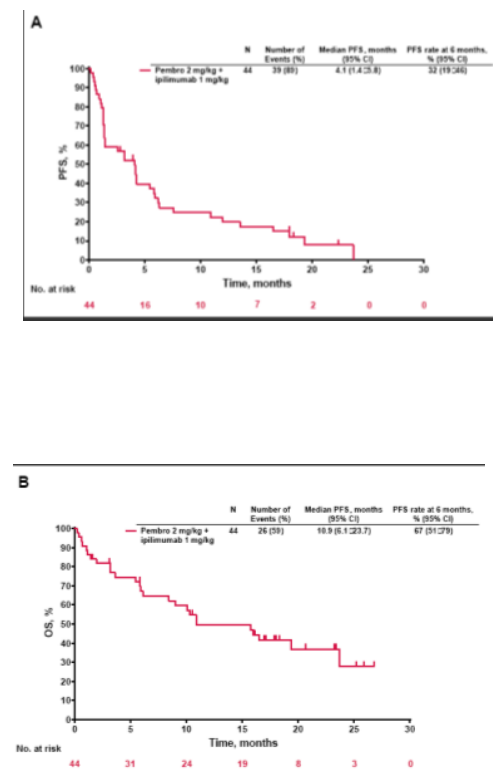
Pembrolizumab Plus Ipilimumab as Second-Line Therapy for Advanced NSCLC

Table 1. Baseline Demographic and Disease Characteristics

\begin{tabular}{|c|c|c|}
\hline & $\begin{array}{c}\text { Pembrolizumab } 10 \mathrm{mg} / \mathrm{kg}+ \\
\text { ipilimumab } \\
(\mathrm{N}=6)^{\mathrm{a}}\end{array}$ & $\begin{array}{c}\text { Pembrolizumab } 2 \mathrm{mg} / \mathrm{kg}+ \\
\text { ipilimumab } 1 \mathrm{mg} / \mathrm{kg} \\
(\mathrm{N}=45)^{\mathrm{b}}\end{array}$ \\
\hline Median (range) age, y & $64(54-75)$ & $61(28-79)$ \\
\hline \multicolumn{3}{|l|}{ Sex, n (\%) } \\
\hline Male & $3(50)$ & $23(51)$ \\
\hline Female & $3(50)$ & $22(49)$ \\
\hline \multicolumn{3}{|l|}{ ECOG performance status, $\mathrm{n}(\%)$} \\
\hline 0 & $1(17)$ & $11(24)$ \\
\hline 1 & $5(83)$ & $34(76)$ \\
\hline Tumor histology, n (\%) & & \\
\hline Adenocarcinoma & $4(67)$ & $37(82)$ \\
\hline Squamous & 0 & $4(9)$ \\
\hline NSCLC not otherwise specified & $2(33)$ & $4(9)$ \\
\hline \multicolumn{3}{|l|}{ Smoking status, n (\%) } \\
\hline Current or former smoker & $4(67)$ & $34(76)$ \\
\hline Never smoker & $2(33)$ & $10(22)$ \\
\hline Missing & 0 & $1(2)$ \\
\hline Brain metastasis, $\mathrm{n}(\%)$ & $1(17)$ & $14(31)$ \\
\hline \multicolumn{3}{|l|}{ PD-L1 TPS, n (\%) } \\
\hline$<1 \%$ & $1(17)$ & $21(47)$ \\
\hline $1 \%-49 \%$ & $1(17)$ & $18(40)$ \\
\hline 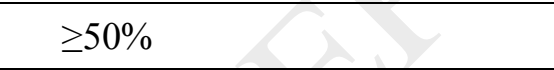 & $4(67)$ & $6(13)$ \\
\hline \multicolumn{3}{|l|}{$\begin{array}{l}\text { Prior lines of systemic therapy, } \mathrm{n} \\
(\%)\end{array}$} \\
\hline 1 & $2(33)$ & $13(29)$ \\
\hline 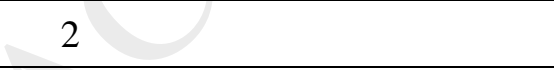 & $4(67)$ & $15(33)$ \\
\hline$\geq 3$ & 0 & $17(38)$ \\
\hline \multicolumn{3}{|l|}{ EGFR mutation status, $\mathrm{n}(\%)$} \\
\hline Wild type & $5(83)$ & $33(73)$ \\
\hline Mutant & $1(17)$ & $10(22)$ \\
\hline Undetermined $^{c}$ & 0 & $2(4)$ \\
\hline
\end{tabular}


Pembrolizumab Plus Ipilimumab as Second-Line Therapy for Advanced NSCLC

\begin{tabular}{|c|c|c|}
\hline & $\begin{array}{c}\text { Pembrolizumab 10 } \mathbf{~ m g} / \mathbf{k g}+ \\
\text { ipilimumab } \\
(\mathbf{N}=\mathbf{6})^{\mathbf{a}}\end{array}$ & $\begin{array}{c}\text { Pembrolizumab 2 } \mathbf{~ m g} / \mathbf{k g}+ \\
\text { ipilimumab 1 mg/kg } \\
(\mathbf{N}=\mathbf{4 5})^{\mathbf{b}}\end{array}$ \\
\hline ALK translocation status, $\mathrm{n}(\%)$ & & \\
\hline Wild type & $5(83)$ & $41(91)$ \\
\hline Mutant & 0 & $1(2)$ \\
\hline Undetermined $^{\mathrm{c}}$ & $1(17)$ & $3(7)$ \\
\hline
\end{tabular}

ECOG, Eastern Cooperative Oncology Group; NSCLC, non-small-cell lung cancer; PD-L1, programmed death ligand 1; TPS, tumor proportion ccore.

${ }^{a}$ Three patients received pembrolizumab $10 \mathrm{mg} / \mathrm{kg}$ plus ipilimumab $1 \mathrm{mg} / \mathrm{kg}$, and 3 patients received pembrolizumab $10 \mathrm{mg} / \mathrm{kg}$ plus ipilimumab $3 \mathrm{mg} / \mathrm{kg}$.

${ }^{b}$ One patient in the pembrolizumab $2 \mathrm{mg} / \mathrm{kg}$ plus ipilimumab $1 \mathrm{mg} / \mathrm{kg}$ group had an original diagnosis of NSCLC that was later discovered to be metastatic melanoma and is excluded from efficacy analyses but included in safety analyses.

${ }^{\mathrm{c}}$ Determination of $E G F R$ mutation or $A L K$ translocation was not required in patients with squamous histology; determination of $A L K$ status was not required for patients with $E G F R$ mutations. Determination of $E G F R$ status was not required for patients with known $K R A S$ mutations. 
Pembrolizumab Plus Ipilimumab as Second-Line Therapy for Advanced NSCLC

Table 2. Summary of Response to Treatment

\begin{tabular}{|c|c|c|}
\hline & $\begin{array}{c}\text { Pembrolizumab } 10 \mathrm{mg} / \mathrm{kg}+ \\
\text { ipilimumab } \\
(\mathrm{N}=6)^{\mathrm{a}}\end{array}$ & $\begin{array}{c}\text { Pembrolizumab } 2 \mathrm{mg} / \mathrm{kg}+ \\
\text { ipilimumab } 1 \mathrm{mg} / \mathrm{kg} \\
(\mathrm{N}=44)^{\mathrm{b}}\end{array}$ \\
\hline \multicolumn{3}{|l|}{ Best overall response, $\mathrm{n}(\%)$} \\
\hline Complete response & 0 & $1(2)$ \\
\hline Partial response & $3(50)$ & $12(27)$ \\
\hline Stable disease & $2(33)$ & $11(25)$ \\
\hline Progressive disease & $1(17)$ & $13(30)$ \\
\hline Not evaluable & 0 & $7(16)$ \\
\hline $\begin{array}{l}\text { Objective response rate, } \%(95 \% \\
\text { CI) }\end{array}$ & $50(12-88)$ & $30(17-45)$ \\
\hline $\begin{array}{l}P \text { value (for objective response } \\
\text { rate }>20 \%)^{c}\end{array}$ & - & 0.0858 \\
\hline \multicolumn{3}{|l|}{$\begin{array}{l}\text { Objective response rate by PD-L1 } \\
\text { TPS status, } \mathrm{n} / \mathrm{N}(\%)^{\mathrm{d}}\end{array}$} \\
\hline PD-L1 TPS < $1 \%$ & $-8 y$ & $5 / 20(25)$ \\
\hline PD-L1 TPS $1 \%-49 \%$ & - & $7 / 18(39)$ \\
\hline PD-L1 TPS $\geq 50 \%$ & - & $1 / 6(17)$ \\
\hline $\begin{array}{l}\text { Time to response, } \\
\text { median (range), mo }\end{array}$ & $1.4(1.4-20.7)$ & $2.7(1.3-6.2)$ \\
\hline $\begin{array}{l}\text { Duration of response, } \\
\text { median (range), mo }\end{array}$ & Not reached $(8.3+$ to $28.8+)$ & $10.5(2.5+$ to 21.0$)$ \\
\hline
\end{tabular}

PD-L1, programmed death ligand 1; TPS, tumor proportion score.

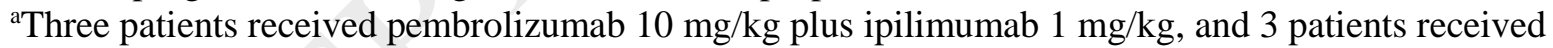
pembrolizumab $10 \mathrm{mg} / \mathrm{kg}$ plus ipilimumab $3 \mathrm{mg} / \mathrm{kg}$.

${ }^{\mathrm{b}}$ One patient in the pembrolizumab $2 \mathrm{mg} / \mathrm{kg}$ plus ipilimumab $1 \mathrm{mg} / \mathrm{kg}$ group had an original diagnosis of NSCLC that was later discovered to be metastatic melanoma and is excluded from efficacy analyses but included in safety analyses.

'One-sided $P$ value based on exact binomial distribution for testing the null hypothesis that the objective response rate was $\leq 20 \%$ vs the alternative hypothesis that objective response rate was $>20 \%$.

${ }^{\mathrm{d}}$ The small number of patients in the pembrolizumab $10 \mathrm{mg} / \mathrm{kg}+$ ipilimumab group precludes meaningful analysis of ORR data by PD-L1 TPS status. 
Pembrolizumab Plus Ipilimumab as Second-Line Therapy for Advanced NSCLC

Table 3. Incidence of Adverse Events

\begin{tabular}{|c|c|c|c|c|}
\hline & \multicolumn{2}{|c|}{$\begin{array}{c}\text { Pembrolizumab } 10 \mathrm{mg} / \mathrm{kg}+ \\
\text { ipilimumab } \\
(\mathrm{N}=6)^{\mathrm{a}} \\
\end{array}$} & \multicolumn{2}{|c|}{$\begin{array}{c}\text { Pembrolizumab } 2 \mathrm{mg} / \mathrm{kg}+ \\
\text { ipilimumab } 1 \mathrm{mg} / \mathrm{kg} \\
(\mathrm{N}=45)^{\mathrm{b}} \\
\end{array}$} \\
\hline \multicolumn{5}{|l|}{ Treatment-related AEs, n (\%) } \\
\hline Any grade & \multicolumn{2}{|c|}{$6(100)$} & \multicolumn{2}{|c|}{$29(64)$} \\
\hline Grade 3-5 AE & \multicolumn{2}{|c|}{$1(17)$} & \multicolumn{2}{|c|}{$13(29)$} \\
\hline Led to discontinuation & \multicolumn{2}{|c|}{$1(17)$} & \multicolumn{2}{|c|}{$9(20)$} \\
\hline Led to death & \multicolumn{2}{|c|}{0} & \multicolumn{2}{|c|}{$1(2)$} \\
\hline $\begin{array}{l}\text { Treatment-related AEs occurring in } \\
\geq 3 \text { patients in the pembrolizumab } \\
2 \mathrm{mg} / \mathrm{kg}+\text { ipilimumab } 1 \mathrm{mg} / \mathrm{kg} \\
\text { group, } \mathrm{n}(\%)\end{array}$ & Any grade & Grade $3-5$ & Any grade & Grade $3-5$ \\
\hline Fatigue & $3(50)$ & 0 & $12(27)$ & $1(2)$ \\
\hline Hypothyroidism & $1(17)$ & 0 & $10(22)$ & 0 \\
\hline Decreased appetite & $2(33)$ & 0 & $5(11)$ & 0 \\
\hline Diarrhea & $2(33)$ & 0 & $5(11)$ & $1(2)$ \\
\hline Pruritus & $1(17)$ & 0 & $5(11)$ & 0 \\
\hline Arthralgia & $1(17)$ & 0 & $3(7)$ & 0 \\
\hline Myalgia & $1(17)$ & 0 & $3(7)$ & 0 \\
\hline Rash & $1(17)$ & 0 & $3(7)$ & 0 \\
\hline Headache & 0 & 0 & $3(7)$ & 0 \\
\hline Nausea & 0 & 0 & $3(7)$ & 0 \\
\hline Mucosal inflammation & 0 & 0 & $3(7)$ & 0 \\
\hline Pneumonitis & 0 & 0 & $3(7)$ & $1(2)$ \\
\hline Thyroiditis & 0 & 0 & $3(7)$ & 0 \\
\hline $\begin{array}{l}\text { Immune mediated AEs and infusion } \\
\text { reactions, } \mathrm{n}(\%)\end{array}$ & Any grade & Grade $3-5$ & Any grade & Grade $3-5$ \\
\hline Hypothyroidism & $1(17)$ & 0 & $11(24)$ & 0 \\
\hline Pneumonitis & $1(17)$ & 0 & $3(7)$ & $1(2)$ \\
\hline Uveitis & $1(17)$ & 0 & 0 & 0 \\
\hline Thyroiditis & 0 & 0 & $4(9)$ & 0 \\
\hline Severe skin reactions & 0 & 0 & $3(7)$ & $3(7)$ \\
\hline Colitis & 0 & 0 & $2(4)$ & $2(4)$ \\
\hline Hyperthyroidism & 0 & 0 & $2(4)$ & 0 \\
\hline
\end{tabular}


Pembrolizumab Plus Ipilimumab as Second-Line Therapy for Advanced NSCLC

\begin{tabular}{|l|c|c|c|c|}
\hline & \multicolumn{2}{|c|}{$\begin{array}{c}\text { Pembrolizumab 10 mg/kg + } \\
\text { ipilimumab } \\
(\mathbf{N = 6})^{\mathbf{a}}\end{array}$} & \multicolumn{2}{c|}{$\begin{array}{c}\text { Pembrolizumab 2 mg/kg + } \\
\text { ipilimumab 1 mg/kg } \\
(\mathbf{N}=\mathbf{4 5})^{\mathbf{b}}\end{array}$} \\
\hline Adrenal insufficiency & 0 & 0 & $1(2)$ & $1(2)$ \\
\hline Myasthenic syndrome & 0 & 0 & $1(2)$ & 0 \\
\hline Pancreatitis & 0 & 0 & $1(2)$ & $1(2)$ \\
\hline Type 1 diabetes mellitus & 0 & 0 & $1(2)$ & $1(2)$ \\
\hline
\end{tabular}

AE, adverse event.

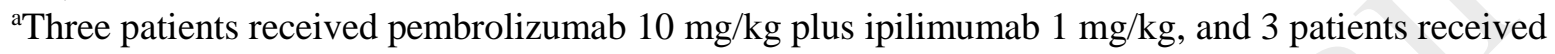
pembrolizumab $10 \mathrm{mg} / \mathrm{kg}$ plus ipilimumab $3 \mathrm{mg} / \mathrm{kg}$.

${ }^{\mathrm{b}}$ One patient in the pembrolizumab $2 \mathrm{mg} / \mathrm{kg}$ plus ipilimumab $1 \mathrm{mg} / \mathrm{kg}$ group had an original diagnosis of NSCLC that was later discovered to be metastatic melanoma and is excluded from efficacy analyses but included in safety analyses. 